\title{
Specific Retrieval Deficit from Long-Term Memory in Children with Poor Arithmetic Facts Abilities
}

\author{
C. Mussolin* and M-P. Noël
}

Department of Cognition and Development, Faculty of Psychology, University of Louvain, Louvain-la-Neuve, Belgium

\begin{abstract}
The most common characteristic of children with mathematical disorders is a difficulty in retrieving basic arithmetic facts. Different hypotheses about the underlying cause and the specificity of this deficit have been postulated. Some of them are general (such as low speed-of-processing or difficulties in retrieving semantic information from longterm memory), others are more specific (such as problems with material requiring verbal routines or a deficit in representing or accessing arithmetic facts). To clarify this, children with poor arithmetic facts abilities (PoorAF) and control children of the same age performed five computerized tasks requiring the retrieval of information from long-term memory such as the product of multiplication facts, words semantically related to a target, objects functionally associated with a target, countries corresponding to a capital city presented as target, and the final word of incomplete French proverbs. In addition, a perceptual task was used as a measure of children's processing speed. It was found that children with PoorAF were significantly slower than control children only in the arithmetic retrieval task. These findings support the hypothesis of a specific numerical deficit altering the retrieval of the arithmetic network from long-term memory.
\end{abstract}

Key Words: Developmental dyscalculia, mathematical disorders, arithmetic development, long-term memory.

\section{INTRODUCTION}

There have been a large number of studies of learning disorders in mathematics in the last ten years. This learning disability that refers to a deficit in the processing of numerical and arithmetic information in the context of otherwise normal academic achievement [1], affects between 5 and $8 \%$ of school-aged children [2-4]. Children with mathematical disorders (MD) tend to display heterogeneous patterns of numerical skills [5-7], but the most consistent finding is their difficulty with simple calculations. In those situations, they produce more errors and their reaction time pattern differs from that of younger children without a learning deficit [810]. This profile is mainly due to their difficulties in retrieving basic arithmetic facts from long-term memory [11-17]. For most children with MD, the ability to retrieve basic facts does not substantively improve during the elementary-school years, suggesting that the retrieval difficulties result from a persistent cognitive deficit, rather than delayed development [13].

Recent theories suggest that the poor retrieval of arithmetic facts by children with MD could come from a deficit in the basic processing of numbers [18]. However, other more general hypotheses have been proposed to account for these difficulties. Such hypotheses refer to deficits in general speed-of-processing, the retrieval of information from semantic memory, or even the retrieval of verbal routines.

The hypothesis of a general deficit in speed-of-processing was supported by the Bull and Johnston's [19] results. They showed that children's arithmetic ability was best

\footnotetext{
*Address correspondence to this author at the Department of Cognition and Development, Faculty of Psychology, University of Louvain, Louvain-laNeuve, Belgium; Tel: +32 (0)10 4790 43; Fax: +32 (0)10 4737 74;

E-mail: christophe.mussolin@uclouvain.be
}

predicted by a general processing speed measure and that children with MD are slow not only in numerical tasks (identifying numbers, executing arithmetic procedures) but also in non-numerical ones (perceptual-motor performance). The authors conclude that slow processing could interfere with the automatic access to basic arithmetic facts in children with MD.

According to Geary and his colleagues [13, 20], difficulties in representing and/or accessing information from semantic memory might contribute to the weakness in retrieving arithmetic facts found in children with MD. This deficit could also explain the high co-occurrence of mathematical and reading disorders [2, 21]. Similarly, Geary, Hamson, and Hoard [22] showed that children with both mathematical and reading difficulties were slow to articulate familiar words, although their performance with non-words was not impaired. This suggests poor activation of phonological or semantic representations. Other support for this hypothesis can be found in the study by Temple and Sherwood [23] who showed that children with MD were slower than control children in a task involving the retrieval of arithmetic facts, as well as in naming line drawings of objects and the colours of patches. However there were only four participants in this study, which prevents any strong conclusion being drawn.

The third hypothesis is more specific as it supposes a deficit in retrieving verbal routines from long-term memory. Indeed, the triple-code model [24] assumes that single-digit multiplications and, to a lesser extent, single-digit additions are usually performed by rote verbal memory retrieval. The operands of the problem are transcoded into a verbal representation which is used to retrieve the correct response using rote verbal memory. By contrast, subtraction and division problems are processed as abstract quantity representations which are used for semantic manipulations and calculation 
procedures ${ }^{1}$. From this prediction, we might expect that children with MD characterized by poor abilities in the retrieval of arithmetic facts, and particularly multiplication facts, would be impaired in other tasks supported by rote verbal memory. Accordingly, Wilson and Dehaene [25] propose various hypotheses for developmental dyscalculia including a verbal memory dyscalculia that would be related to difficulties in learning and retrieving arithmetic facts, particularly multiplication, and possibly the counting sequence as well.

In addition to these hypotheses postulating a general deficit, it has been suggested that children with MD have a very specific ${ }^{2}$ deficit in their capacity to represent and manipulate numbers. Butterworth [18] argues that we are born with an innate capacity for recognising and mentally manipulating numerosities, and that this capacity is embodied in specialized neural circuits. A large body of evidence indicates that semantic memory for numbers is mediated by a different system to general semantic memory [26, 27]. In this context, developmental dyscalculia corresponds to a highly selective and specific deficit of a basic capacity for understanding numbers, which leads to a range of difficulties in learning about numbers and arithmetic. A very similar hypothesis has been proposed by Dehaene [28] who postulates a deficit of the "number sense" corresponding to our ability to quickly understand, approximate and manipulate numerical quantities.

Recent behavioural work has shown that children with MD perform worse than control children on tasks involving processing numbers, even very basic tasks such as counting or comparing the magnitude of two numbers [29-32]. The intraparietal sulcus could be the neurofunctional correlate of this specialized number system because it is consistently activated in number comparison [33-35], and in arithmetic tasks [36]. Furthermore, this region has been found to be disturbed in people with MD [37, 38].

Here, we are not interested in providing additional evidence that children with MD have problems in basic number processing. Rather, the purpose of the present study is to examine whether the difficulty in retrieving arithmetic facts from long-term memory is specific. To this end, children showing poor multiplication facts abilities are compared to children without any learning disability on six computerized selection tasks. Five of them required the child to retrieve information from long-term memory, while the child had to make perceptual associations in the last task. These tasks were specifically developed to test the four hypotheses mentioned above (i.e., a general problem of processing speed, a general deficit in retrieving information from se-

\footnotetext{
${ }^{1}$ These postulates are based on the examination of two acalculic patients. Patient BOO suffered from a left subcortical lesion and was impaired in all tasks requiring rote verbal retrieval, including but not limited to arithmetic tables; her semantic system of numerical quantities was intact. In contrast, patient MAR displayed clinical features of Gerstmann's syndrome (Gerstmann, 1940) with a lesion in the inferior parietal lobule, and was impaired in tasks requiring the manipulation of semantic numerical knowledge (such as number bisection and subtraction), although the retrieval of rote arithmetic facts was preserved.

${ }^{2}$ We use the term "specific" through this paper to indicate that the difficulties of children with PoorAF impaired the retrieval of arithmetic facts from long-term memory, but not the retrieval of other kind of information. It doesn't mean that these children had no problem with other mathematical skills as numerical estimation, for example.
}

mantic memory, difficulty in the retrieval of verbal routines, and a specific deficit restricted to the retrieval of arithmetic facts).

To assess whether children had a slow processing speed in general, we developed a perceptual task that did not require any memory retrieval: one shape was presented as a target and the child had to decide which of two other shapes was identical to the target. A possible deficit in retrieving semantic information from long-term memory was evaluated using three tasks. In the first, a target word was presented. The child had to select from two other words, the one that was semantically related to the target. The second task was the same, except that non-symbolic material was used: words were replaced by line drawings of objects. The third task involved the retrieval of more abstract and arbitrary associations: the name of a capital city was presented as a target and the child had to select the name of the corresponding country from two alternatives. To assess a possible difficulty in retrieving verbal routines from memory, French expressions (proverbs) were presented to the children who had to select, from two alternatives, the word that correctly completed the proverb. Finally, a task testing the retrieval of arithmetic facts was used: multiplication problems were presented to the children who had to select the correct answer from two alternatives. Multiplication facts were presented because this operation elicits an automatic retrieval from long-term memory [39-41].

The predictions are clear. If the difficulty in retrieving simple arithmetic facts and, more specifically, multiplication facts is due to a general deficit in processing speed, then slow responses in the multiplication task should be associated with slow responses in all the other tasks. If it is due to a global problem in retrieving information from semantic memory, then it should be associated with weak performance on the four tasks that involve such retrieval, i.e., association of words, association of line drawings of objects, association of capital cities and countries, and retrieval of verbal proverbs. If it is due to a deficit in retrieving verbal routines, then only the retrieval of verbal proverbs should be impaired in children with weak multiplication skills. Finally, if the learning disability in arithmetic is a very specific problem restricted to the number domain, then children with low ability in multiplication should score normally on all the nonnumerical tasks.

\section{MATERIALS AND METHODOLOGY}

\section{Participants}

A total of 125 fourth-grade children (63 boys, 62 girls; mean age $=116$ months, $\mathrm{SD}=5.7$ months) from three schools in Belgium performed an achievement test in calculation (i.e., a multiplication fluency test). Parental consent was obtained for all of them. From this population, 18 children were selected to constitute the group of poor arithmetic facts abilities. For each of these children, a control child without any learning difficulties was picked from the same class, matched as closely as possible for age, gender, and IQ.

\section{Classification Scheme}

To select the experimental group, the children performed a multiplication fluency task in which they were required to 
solve as many simple multiplications as possible in two minutes. Problems including operands from 1 to 9 were presented to the participants, who were instructed to calculate and write down the correct response as quickly as possible. The problems had to be attempted in the order in which they were presented. The Similarities and the Images Completion subtests of the WISC-III [42] were also administrated to the children, to provide an estimate of their IQ scores, as suggested by Sattler [43]. Children's reading abilities were assessed by two standardised tests: the LUM and the L3. The LUM [44] is a speeded reading test in which the child reads aloud as many words as possible from a printed protocol in 60 seconds. The $\mathrm{L} 3[45,46]$ is a reading comprehension test in which the child must select the correct word from various alternative answers that are semantically related to the sentences, with a 5 minutes time limit.

Two groups were constituted. The first group included 18 children who performed poorly in the test of retrieval of arithmetic facts (PoorAF group). They all scored below the $15^{\text {th }}$ percentile on the multiplication fluency test ( 10 boys, 8 girls; mean age $=117$ months), but were within one standard deviation of the mean of the normative data for the two reading achievement tests. This is a more stringent criterion than that used in previous studies (the $30^{\text {th }}$ percentile, [47]; or the $35^{\text {th }}$ percentile, [6]). The second group consisted of 18 academically normal children (Control group) with mean achievement scores at or above the $50^{\text {th }}$ percentile on the calculation test, and reading scores within one standard deviation of the mean of the normative data for both LUM and L3 tests ( 10 boys, 8 girls; mean age $=118$ months $)$.

For each group, the numbers of boys and girls, mean age, IQ, and achievement scores are shown in Table 1. An independent $t$-test revealed a significant group effect on the calculation achievement test $(t(1,34)=12.529, p<.001)$. The mean ages did not differ significantly between the groups $(t$ $<1)$, nor did the number of boys and girls $\left(\chi^{2}(1)=0.22, p>\right.$ $.05)$. Neither were there any significant differences between the two groups on their scores on the two IQ subtests or their estimated IQ scores (all, $t<1$ ). Groups differences on the
LUM reading test, $(t(1,34)=1.911, p=.064)$ and the L3 comprehension test $(t(1,34)=1.797, p=.081)$ were marginally significant. These variables were therefore entered as covariates into the subsequent analyses of variance (ANOVA). However, as described later in the RT analyses, controlling for reading ability was necessary in the word selection task only. Indeed, the performances in the word selection task were closely related to LUM scores. The other selection tasks yielded similar results irrespective of whether LUM or L3 was entered or not as covariate.

\section{Experimental Tasks and Procedure}

The experiment was displayed on a PC running E-Prime software, version 1.1. As illustrated in Fig. (1), children performed six different experimental tasks. Each task consisted of the presentation of three visual stimuli on the screen (one target and two propositions). A fixation point was first flashed for $100 \mathrm{~ms}$ and was replaced by the target followed after $150 \mathrm{~ms}$ by the two possible answers. Both target and answers remained on the screen until the child responded. The interstimulus interval was fixed at $500 \mathrm{~ms}$. Participants were instructed to press the response key corresponding to the position of the correct proposition. Each task was presented in a block with a pause in-between. The stimuli were ordered randomly within each block. The experiment started with the presentation of the multiplication selection task, but the order of the other tasks was counterbalanced across participants.

\section{Multiplication Selection Task}

The retrieval of arithmetic facts was assessed using a forced choice multiplication task. Forty single-digit multiplications with operands between 2 and 9 (except ties problems) appeared on the screen with two possible answers. The wrong answer had the same parity as the correct result, thus preventing the use of a short-cut based on parity checking [48]. The two answers always had the same decade digit. Half of the distracters was operand-related. As in previous studies investigating performance on arithmetic tasks, we

Table 1. Mean and Standard Deviation of the Age, Multiplication Fluency Test Score, Images Completion and Similarities IQ Subtests, LUM and L3 Reading Tests, for the PoorAF and Control Groups

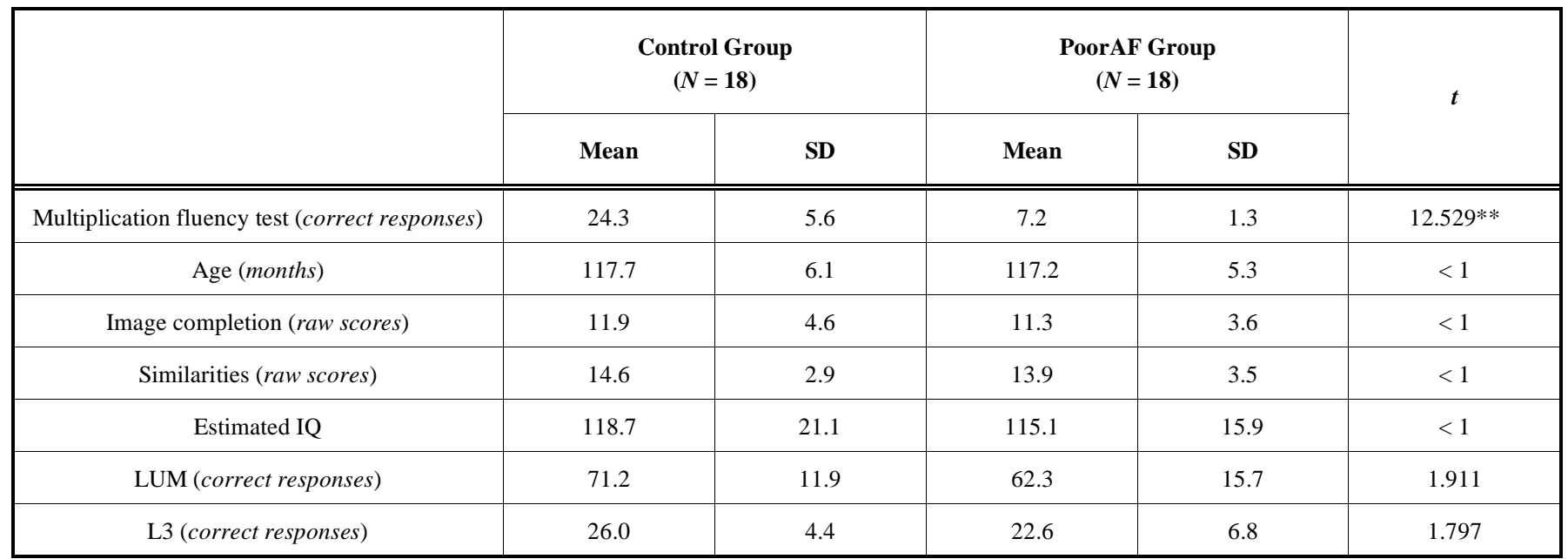

$* p<.05 ; * *<<.001$. 


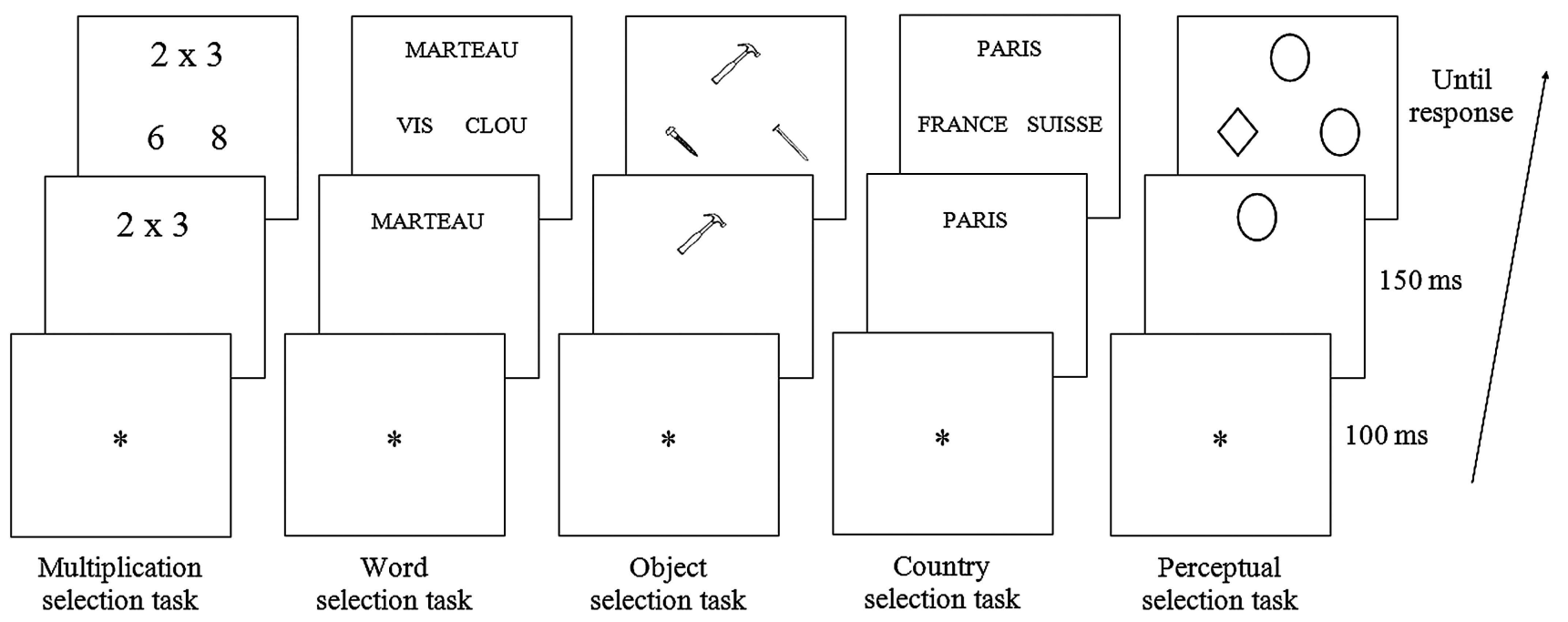

Fig. (1). Examples of stimuli used for each of the six selection tasks: multiplication problems, words ('marteau-vis-clou' in French, 'hammer-screw-nail' in English), object, capitals and countries ('Paris-France-Suisse' in French, 'Paris-France-Switzerland' in English), proverbs ('la chair de poule' in French, 'goose-skin' in English), and perceptual associations. The fixation point was first presented for $100 \mathrm{~ms}$, and was then replaced by the target. The two response propositions appeared followed $150 \mathrm{~ms}$ and remained on the screen until the child made a decision.

analyzed the problem-size effect reflecting the organisation of the arithmetic facts network. The problem-size effect refers to an increase in response times (RTs) and error rates as the size of numbers increases. Many explanations for this effect have been suggested, including the search distance in the fact network [39, 49], the familiarity [50], and the strength of association between the problem and the correct answer [41, 51, 52]. Two problem sizes were defined: in small problems, the two operands were both equal to or below 5 (e.g., $2 \times 3$ ), whereas for medium problems one of the two operands was always above 5 and one was equal to or below 5 (e.g., $7 \times 4$ ). Large problems (where both operands were above 5) were not included, to favour the use of the strategy of retrieval from long-term memory. Although we cannot reject the possibility that children relied on procedural strategies to perform the task, it has previously been shown that both 10-11-year-old children and adults use direct retrieval from memory to access small-multiplication facts [53].

\section{Word Selection Task}

High-frequency written words (selected from [54]) were used to generate 20 associations in the word selection task. Two alternative responses were chosen which both belonged to the same semantic category, although only one was functionally related to the target. Within each pair, the distracter and the target were matched for frequency $(t<1)$, number of letters $(t(1,38)=1.067, p>.05)$, and number of phonemes $(t(1,38)=1.356, p>.05)$. The children were asked to choose the word functionally associated with the target.

\section{Object Selection Task}

Twenty line drawings of concrete objects selected from Snodgrass and Vanderwart [54] were used in the object selection task. These stimuli corresponded to the words in the previous selection task. In each trial, the two objects presented as probes not only had a semantic relation to each other, but were also structurally close. Here again, the task was to choose the object functionally related to the target.

\section{Country Selection Task}

To assess the retrieval of arbitrarily learned information from long-term memory, the name of a capital city was presented followed by the names of two European countries. The two possible answers had, as far as possible, the same number of letters $(t<1)$. Twenty associations were presented in which participants had to select the country corresponding to the capital city.

\section{Proverb Selection Task}

Children were asked to complete 20 simple French proverbs, all composed of four words. The three first words of each proverb were presented as the target with two possible final words. The distracter and the target were always in the same semantic category to avoid decisions based on relevant attributes. In each trial, the two possible responses were matched for the number of letters, the frequency of the written words, and the number of phonemes (all, $t<1$ ).

\section{Perceptual Selection Task}

In this task, the children were presented with two shapes and asked to choose the one that was identical to the target. Through 20 perceptual associations, this task allowed us to obtain a measure of processing and decision speed for children with PoorAF and control children.

Before each task, the children were presented with a training list of 5 associations randomly selected from the experimental set. This enabled them to practice the task and to familiarized themselves with the procedure. Data from these trials were not included in the analyses. Children were asked to do the tasks as well as they could, but were assured that their performance would not be graded for school assessment. The instructions emphasized both speed and accuracy. Each session lasted approximately $20 \mathrm{~min}$.

\section{Procedure}

Three testing sessions were conducted at the end of the academic year. The first collective session was dedicated to 


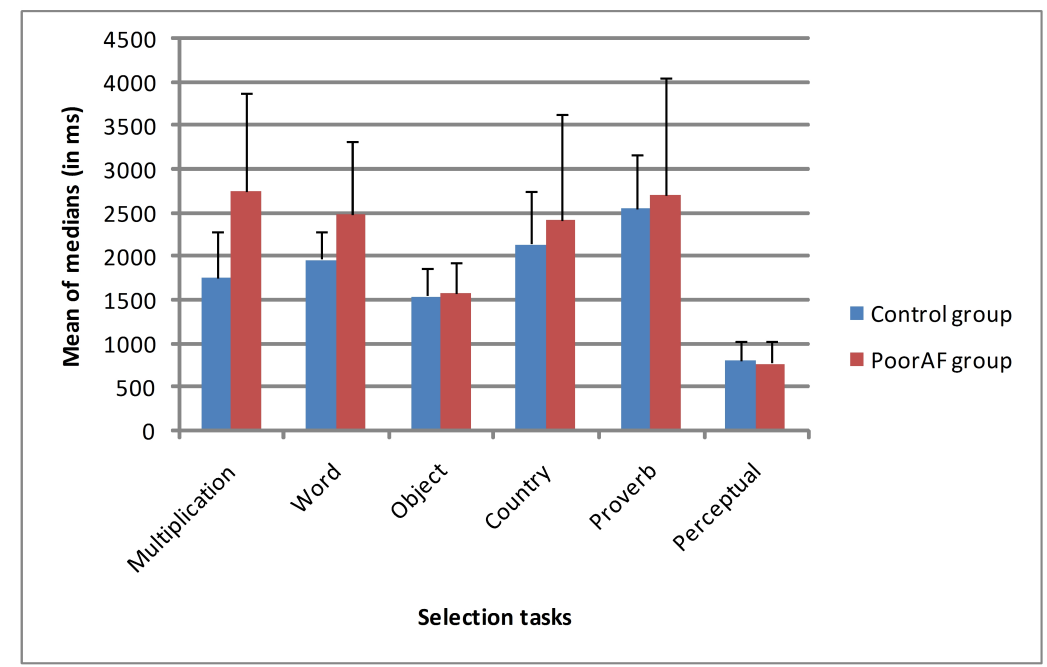

Fig. (2). Mean of medians RTs on the six selection tasks for the PoorAF and control children.

the initial selection (i.e., to the mathematical assessment). On the basis of these results, the IQ subtests and the two reading achievement tests were administrated to the selected children in the second session. The tests were presented in the following order: LUM, L3, Similarities, and Image Completion. Finally, the experimental tasks described above were performed in the third session. The order of the tasks was counterbalanced across participants.

\section{RESULTS}

\section{Error Analyses}

The error rate for each task was calculated for every participant separately, and entered into a mixed ANCOVA with group as the between-subjects factor, tasks as the withinsubjects factors, and LUM scores as the covariate. The main effect of task was significant in the analysis $(F(5,165)=$ $12.142, p<.001)$. The perceptual selection task yielded the lowest average error rate $(2.6 \%)$, followed by the object selection task $(4.9 \%)$, the multiplication selection task $(6.5 \%)$, the word selection task $(7.1 \%)$, the country selection task $(29.3 \%)$, and finally the proverb selection task (30.9\%). Post hoc $t$-test analyses indicated that there were no significant differences between the selection tasks with forms, pictures, multiplications, and words, but that these tasks differed significantly from the country selection task $(p<.001)$ and the proverb selection task $(p<.001)$, which did not differ from each other. The group effect was not significant $(F<1)$ and did not interact with the task $(p>.05)^{3}$.

We also investigated the effect of problem size in the multiplication selection task. In an ANOVA with problem size and group, the results indicated a main effect of problem size, $(F(1,34)=7.531, p=.01)$, indicating higher error rates for medium multiplication facts $(8.3 \%)$ than for small multiplication facts $(4.7 \%)$. The group was not significant and did not modulate the problem size effect (all, $F<1$ ). Importantly, the error analysis indicated that the multiplication problems

\footnotetext{
${ }^{3}$ A similar ANCOVA was conducted with the L3 scores as covariate. This analysis yielded the same results with task being the only significant effect $(F(5,165)=10.690$,
} $p<.001)$. used in the present study were not too difficult for the children with PoorAF as their error rates were low and comparable to those of the control children.

\section{RT Analyses}

A first 2 (group) by 6 (task) mixed ANCOVA, with group as the between-subjects factor, tasks as the withinsubjects factors, and LUM scores as the covariate, was carried out on RTs for correct trials only ${ }^{4}$. The analysis revealed a main effect of task $(F(5,165)=3.480, p=.005)$, indicating that the perceptual selection task $(798 \mathrm{~ms})$ was performed fastest, followed by the object selection task $(1567 \mathrm{~ms})$, the multiplication selection task (2261 ms), the word selection task (2225 ms), the country selection task (2284 ms), and finally the proverb selection task $(2633 \mathrm{~ms})$. Post hoc $t$-test analyses showed that the RTs for the perceptual selection task differed significantly from the others $(p<.001)$, and that there was also a significant difference between the object selection task and the other four tasks $(p<.001)$, which did not differ from each other.

More importantly, the group effect was not significant ( $p$ $>.05)$ but interacted with the task $(F(5,165)=2.423, p=$ .038). As illustrated in Fig. (2), separate analyses for each task revealed that the effect of group was only significant on the multiplication selection $\operatorname{task}^{5}(F(1,33)=7.925, p=$ $.008)$. The PoorAF group had slower average RTs (2762 ms) than the control group (1759 ms). Although the children with PoorAF were also slower on the selection tasks with countries and proverbs, the differences were not significant (all,

\footnotetext{
${ }^{4}$ The RT analyses reported below were also carried out on z-scores and yielded the same results with the group effect being significant only for the multiplication task when controlled for reading ability $(F(1,33)=8.220, p<.007)$.

${ }^{5}$ To ensure that the lack of group effect on the country and proverbs selection tasks was not due to their high error rates, one-way ANOVAs were conducted for the children who scored well above the chance rate (i.e., had scores of at least $15 / 20, \chi^{2}(1)=5$, $p<.05)$. For the country selection task, the 11 children with MD who scored at or above the criterion did not differ from the 8 control children $(F<1$, medians of 2245 $\mathrm{ms}$ and $2042 \mathrm{~ms}$ respectively). Similarly, no significant difference was found on the proverb selection task between the 9 control and $8 \mathrm{MD}$ children who reached the criterion level, $F(1,16)=2.49, p>.05(2405 \mathrm{~ms}$ for controls and $2997 \mathrm{~ms}$ for MD children).
} 
$p>.05)^{6}$. The difference observed with words was significant when the children were not controlled for their reading ability $(F(1,33)=5.705, p=.023)$, but disappeared when LUM or L3 scores were introduced as a covariate.

For the multiplication selection task, an ANOVA was conducted with problem size and group. The main effects of group $(F(1,34)=37.307, p<.001)$ and problem size $(F(1$, $34)=12.372, p=.001$ ) were both highly significant. Children in the PoorAF group were slower than control children (2914 ms and $1822 \mathrm{~ms}$, respectively) and small multiplications were processed faster $(1957 \mathrm{~ms})$ than medium ones (2779 ms). Interestingly, the interaction between the two effects was marginally significant $(F(1,34)=4.016, p=$ $.053)$, reflecting a stronger influence of problem size on children in the PoorAF group (difference of $1091 \mathrm{~ms}$ between medium and small multiplications) than on children in the control group (difference of $553 \mathrm{~ms}$ ).

To further investigate the independency of the multiplication task relative to the other selection tasks, we conducted a factor analysis on the median RTs of the global children population. Two significant components were extracted by the analysis. The first component accounted for $57 \%$ of variance and the second component explained $20 \%$ of variance. The component matrix before rotation indicated that the multiplication selection task had strong saturations on the second component (.75) while the other selection tasks exhibited saturations on the first component (from .62 to .85). We used quartimax rotation in an attempt to maximize the spread of factor loadings for a variable across all factors, making the interpretation easier. The component matrix after rotation confirmed these results showing that the multiplication task had saturations on the second component only. By contrast, the selection tasks including objects, proverbs, countries, and forms showed more saturation on the first component. The interpretation for the words selection task was more difficult because this task presented saturations on both components (.64 for the first and .63 for the second component).

\section{DISCUSSION}

It is now well established that developmental dyscalculia is associated with problems in retrieving arithmetic facts from long-term memory $[8,10]$. Several hypotheses have been proposed to account for this deficit. Some of them are very general, such as the hypothesis of a low processing speed [19] or of a global retrieval deficit of information from semantic memory $[20,22]$. Others are more specific, such as the hypothesis of a deficit in retrieving verbal routines from long-term memory [24], or the hypothesis of a number system deficit [18]. The goal of the present study was to disentangle these four hypotheses by contrasting the performance on several speeded tasks, of children who performed poorly vs. normally on a simple multiplication task.

The results showed that the deficit in retrieving multiplication facts was not associated with a global low processing

\footnotetext{
${ }^{6}$ We conducted a second ANCOVA with group as the between-subjects factor, tasks as within-subjects factors, and L3 scores as the covariate. The analysis showed similar results, with a main effect of task $(F(5,165)=6.810, p<.001)$, and a task $\times$ group interaction, $(F(5,165)=4.502, p=.001)$. Further analyses by task indicated a group effect for the selection task with multiplication problems $(F(1,33)=11.695, p=.002)$, but not for the other tasks $(p>.05)$
}

speed as the two groups tested did not differ in a task were they had to match two shapes. Nor do our findings support the predictions of the theory of a general deficit in retrieving information from semantic memory. Indeed, the children who performed poorly and normally on the multiplication fluency task did not differ on the three tasks requiring them to retrieve information from long-term semantic memory. They scored similarly when asked to select the word or the object associated with a target and when asked to select the country corresponding to the target capital name. Finally, difficulties in retrieving multiplication facts could not be explained by a deficit in retrieving rote verbal materials. The selection task involving proverbs was more difficult than the other tasks for both groups of children, but there were no significant differences between the two groups.

Table 2. Component Matrix after Rotation from the Factor Analysis

\begin{tabular}{|c|c|c|}
\hline \multirow{2}{*}{ Tasks } & \multicolumn{2}{|c|}{ Component } \\
\cline { 2 - 3 } & $\mathbf{1}$ & $\mathbf{2}$ \\
\hline \hline Multiplication & & .86 \\
\hline Word & .64 & .63 \\
\hline Object & .85 & \\
\hline Proverb & .83 & \\
\hline Country & .79 & \\
\hline Form & .78 & \\
\hline
\end{tabular}

Note: Loadings less than .4 are not presented.

Our study indicated that children performing poorly on the multiplication fluency task were significantly slower than their normal peers on the arithmetic task only. They needed about one second more than control children to recognize the correct product related to multiplication problems, but had comparable latencies on the other tasks. These results fit well with "the defective number module hypothesis" in which Butterworth [18] argues that a highly selective and specific deficit for understanding the concept of numerosity leads to general problems in manipulating numbers. Indeed, it seems that children with MD perform poorly in very simple tasks such as counting [55] or comparing numerical magnitudes $[22,30,32]$. In the course of their development, the weak basic numerical abilities of children with MD could alter the learning of arithmetic facts and rules that, in turn, cause difficulties in retrieving arithmetic facts from long-term memory. A specific deficit altering the processing or representation of numerosities might more adequately account for the difficulties encountered by children with MD. In line with this view, neuroimaging studies showed that the intraparietal sulcus which is supposed to hold an abstract representation of number magnitude [56] is consistently activated in calculation $[36,57]$ and was found to be impaired in people with MD [37, 38], suggesting an important role of this number magnitude representation for more elaborated processes. Recently, Wilson and Dehaene [25] reviewed the possible causes for developmental dyscalculia and point out promis- 
ing preliminary evidence for a core deficit of "number sense" which corresponds to our ability to quickly understand, approximate and manipulate numerical quantities [28]. The authors propose that although a majority of children might suffer from a core impairment in number sense, other candidates for dyscalculia are likely to co-exist. In particular, it is plausible to find a double dissociation between a "number sense" dyscalculia vs. a "verbal memory" dyscalculia as observed in adult patients with acalculia [24, 58]. Contrarily to the number sense acalculia in which the patient has lost the sense of the meaning of numbers resulting in difficulties to perform number comparisons and subtraction problems, the verbal memory acalculia refers to an intact understanding of the meaning of numbers but an impairment in retrieving from memory basic multiplication or addition facts. However, there are only a few developmental dyscalculia cases reported in the literature [59-61], and none of them support the verbal memory deficit. Rather, performances of dyscalculic patients favour the number sense deficit. For example, the case of Charles [18], a young dyscalculic adult who despite normal IQ and reasoning, shows difficulties in basic numerical abilities as number comparison. In fact, whereas such dissociation was found in adults with acalculia, the same pattern is not necessary expected in young children who first manipulate the number sense to constitute a wellformed network of arithmetic facts before to retrieve them from long-term memory by verbal routines. More research is needed to investigate the hypothesis of different subtypes of developmental dyscalculia.

While the present findings support a basic deficit of number processing in MD, previous works suggest different hypotheses. Bull and Johnston [19] tested the hypothesis of general processing speed deficit on 7 years old children placed into high- or low-ability group according to an arbitrary criterion based on their mathematics standard age score. The low-ability group showed poorer performance on speed of number identification and visual number matching task, but not on letter identification or processing speed task using geometric figures. Thus, the fact that children with lowability in mathematics were slower than high-ability children in tasks implicating symbolic quantities but not other kind of materials could stem from a specific difficulty with numerical information rather than a general processing speed deficit. The distinction between rote verbal memory and abstract quantity representations on arithmetic facts knowledge is essentially based on the examination of brain-damaged patients $[62,63]$ and supported by works in bilinguals people [64]. However, neuroimaging studies on healthy adults failed to find activation of classical language-based brain regions in arithmetic facts retrieval $[33,65]$. Some works found an influence of angular gyrus in calculation [66, 67], but corresponding to deactivation of this region in reference to the baseline condition. Finally, there is little empirical evidence for a general semantic deficit in children with MD, as argued by Geary and colleagues $[13,20]$. None of the studies investigating this hypothesis yielded very conclusive results. In addition, because reading and mathematics problems are dissociable, it is difficult to postulate that the same deficit can account for the two kinds of learning disabilities.

More generally, it is clear that performance in each selection task depended on the nature of the information to retrieve from long-term memory. In particular, the association power between the target and the correct response, or between the target and the distracter are specific to the cognitive domain tested. Therefore, difficulties observed in the calculation task could reflect a more general problem with interference. However, this hypothesis is very unlikely because no other task was affected by this slowing down. Another point is that our selection tasks did not possess the same discriminating power. Consequently, it is possible that the perceptual task was too easy for children to find group differences. Similarly, the selection task with proverbs could be too difficult to point out a slowing down in children with PoorAF. Nevertheless, performances of control children in the multiplication, word, and object selection tasks were relatively similar and did not differ significantly from each other, suggesting an identical discriminating power. Since children with PoorAF were slower than their control peers in the multiplication task but not in the two other selection tasks, we propose that the discriminating power of the tasks alone can not explain our findings. It is important to note that although this study allowed us to exclude some hypotheses about the difficulties encountered by children with MD, and support a specific deficit, it is still open to debate whether these children really used retrieval strategies to perform the arithmetic task (rather than other strategies such as counting). Children with MD could have difficulty accessing a well-formed network of arithmetic facts (reflecting access deficit), or they could only have a partly stored network of facts (reflecting imperfect storage). Although some theoretical proposals have been made to disentangle a storage and an access deficit [68], such a distinction seems nevertheless to be complicated within the calculation disorders domain. In a case study, Kaufman, Lochy, Drexler, and Semenza [69] tried to clarify the nature of fact retrieval difficulties in a young dyscalculic man. They used behavioural effects reflecting the arithmetic facts network as well as various tasks of production and verification, but were unable to specify the difficulties of their patient and concluded that he exhibited both deficits. A crucial issue for further investigation is the extent to which the pattern presented by children with MD reflects an access deficit, a storage deficit, or both.

To conclude, we would like to emphasize the complexity of the domain of mathematics resulting in a wide array of disabilities. Importantly, the slowness in retrieving arithmetic facts was found here in children with MD characterized by weakness in multiplication facts retrieval and should not be interpreted as a common difficulty for all kinds of learning disabilities in mathematics. Similarly, the question of whether the same deficit accounts for the behaviour of children with MD only, and children with difficulties in both reading and mathematics, remains open. According to Robinson, Menchetti, and Torgesen [70], weak phonological processing abilities could underlie the learning difficulties of children with reading and mathematical disabilities, while children with MD only might be characterized by a weak number sense. Up to now, however, behavioural studies have failed to show clear differences in mathematical performance between the two groups, except for problem resolution $[5,6]$.

\section{CONCLUSIONS}

This paper presents the first experimental evidence of a specific deficit in numerical retrieval from long-term memory in children with MD. Our results add support to a grow- 
ing body of data showing that number processing is mediated by a specific semantic system, different from other semantic systems. Crucially, the difficulties in the retrieval of numbers and arithmetic facts encountered by children with MD could not be attributed to a general deficit in speed of access or to a deficit with verbal material in general, but seem to be specific to the semantic system of numbers.

\section{ACKNOWLEDGEMENTS}

The first author is supported by a grant from the Catholic University of Louvain (FSR), the second author is supported by the National Research Fund of Belgium. The research was also supported by a FRSM fund (fund for scientific and medical research, convention 3.4607.04 F), a concerted research action from the French community of Belgium (ARC 05/10-327), and a Marie Curie Research Training Networks grant from the EEC (MRTN-CT-2003-504927, Numbra project). We are grateful to all our sponsors. Correspondence concerning this article should be addressed to Christophe Mussolin, Department of Psychology, Catholic University of Louvain, Louvain-la-Neuve, Belgium. E-mail: christophe.mussolin@uclouvain.be.

\section{REFERENCES}

[1] Association AP. Diagnotic and Statistical Manual of Mental Disorders. 4th ed. 1994, Washington, DC: American Psychiatric Association.

[2] Badian NA, In: Progress in learning disabilities, Dyscalculia and nonverbal disorders of learning, Myklebust HR, Ed, Grune \& Stratton: New York. 1983; 235-264.

[3] Gross-Tsur V, Manor O, Shalev RS. Developmental dyscalculia: Prevalence and demographic features. Dev Med Child Neurol 1996; 38: 25-33.

[4] Kosc L. Developmental Dyscalculia. J Learn Dis 1974; 7: 164-177.

[5] Hanich LB, Jordan NC, Kaplan D, Dick J. Performance across different areas of mathematical cognition in children with learning difficulties. J Edu Psychol 2001; 93(3): 615-626.

[6] Jordan NC, Hanich LB, Kaplan D. A longitudinal study of mathematical competencies in children with specific mathematics difficulties versus children with comorbid mathematics and reading difficulties. Child Dev 2003; 74(3): 834-850.

[7] Dowker A, In: The development of mathematical skills, Individual differences in normal arithmetical development., Donlan C, Ed, Psychology Press: Hove 1999; 275-302.

[8] Fayol M, Barrouillet P, Marinthe C. Predicting arithmetical achievement from neuropsychological performance: a longitudinal study. Cognition 1998; 68(2): B63-B70.

[9] Russell RL, Ginsburg HP. Cognitive analysis of children's mathematics difficulties. Cog Instruct 1984; 1(2): 217-244.

[10] Raësaënen P, Ahonen T. Arithmetic disabilities with and without reading difficulties: A comparison of arithmetic errors. Dev Neuropsychol 1995; 11: 275-295.

[11] Barrouillet P, Fayol M, Lathulière E. Selecting between competitors in multiplication tasks : An explanation of the errors produced by adolescents with learning difficulties. Int J Behavl Dev 1997; 21: 253-275.

[12] Geary DC. A componential analysis of an early learning deficit in mathematics. J Exp Child Psychol 1990; 49: 363-383.

[13] Geary DC. Mathematical disabilities: Cognitive, neuropsychological, and genetic components. Psycholl Bullet 1993; 114: 345-362.

[14] Jordan NC, Montani TO. Cognitive arithmetic and problem solving: A comparison of children with specific and general mathematics difficulties. J Learn Disabil 1997; 30(6): 624-634.

[15] Ostad SA. Developmental differences in addition strategies: A comparison of mathematically disabled and mathematically normal children. B J Edu Psychol 1997; 67: 345-357.

[16] Garnett K, Fleischner JE. Automatization and basic fact performance of normal and learning disabled children. Learn Disabil Quart 1983; 6: 223-230.
[17] Ostad SA. Cognitive subtraction in a developmental perspective: Accuracy, speed-of-processing and strategy-use differences in normal and mathematically disabled children. Focus Learn Prob Math 2000; 22: 18-31.

[18] Butterworth B. The Mathematical Brain. 1999, London: Macmillan.

[19] Bull R, Johnston RS. Children's arithmetical difficulties: Contributions from processing speed, item identification, and short-term memory. J Exp Child Psychol 1997; 65: 1-24.

[20] Geary DC, Hoard MK. Numerical and arithmetical deficits in learning-disabled children: Relation to dyscalculia and dyslexia. Aphasiology 2001; 15(7): 635-647.

[21] Lewis C, Hitch GJ, Walker P. The prevalence of specific arithmetic difficulties and specific reading difficulties in 9- and 10-year-old boys and girls. J Child Psychol Psychiat 1994; 35: 283-292.

[22] Geary DC, Hamson CO, Hoard MK. Numerical and arithmetical cognition: A longitudinal study of process and concept deficits in children with learning disability. J Exp Child Psychol 2000; 77(3): 236-263.

[23] Temple CM, Sherwood S. Representation and retrieval of arithmetical facts: Developmental difficulties. Quart J Exp Psychol 2002; 55A(3): 733-752.

[24] Dehaene S, Cohen L. Cerebral pathways for calculation: Double dissociation between rote verbal and quantitative knowledge of arithmetic. Cortex 1997; 33: 219-250.

[25] Wilson A, Dehaene S, In: Human behavior, learning, and the developing brain: Atypical development. Number sense and developmental dyscalculia. Coch GDD, Fischer K, Ed, Guilford Press: New York. in press.

[26] Cappelletti M, Butterworth B, Kopelman M. Spared numerical abilities in a case of semantic dementia. . Neuropsychologia 2001; 39: 1224-1239.

[27] Thioux M, Pesenti M, Costes N, De Volder A, Seron X. Taskindependent semantic activation for numbers and animals. Cog Brain Res 2005; 24(2): 284-290.

[28] Dehaene S, In: The number sense, Our numerical heritage: The adult number line. Dehaene S, Ed, UK: Oxford Univ. Press: Oxford. 1997; 64-90.

[29] Bachot J, Gevers W, Fias W, Roeyers H. Number sense in children with visuospatial disabilities: orientation of the mental number line. Spe Iss Psychol Sci 2005; 47(1).

[30] Landerl K, Bevan A, Butterworth B. Developmental dyscalculia and basic numerical capacities: a study of 8-9-year-old students. Cognition 2004; 93(2): 99-125.

[31] Rubinsten O, Henik A. Automatic activation of internal magnitudes: A study of developmental dyscalculia. Neuropsychology 2005; 19(5): 641-648.

[32] Rousselle L, Noël MP. Basic numerical skills in children with mathematics learning disabilities: A comparison of symbolic vs non-symbolic number magnitude processing. Cognition 2007; 102(3): 361-395.

[33] Pesenti M, Thioux M, Seron X, De Volder A. Neuroanatomical substrates of Arabic number processing, numerical comparison, and simple addition: A PET study. J Cognit Neurosci 2000; 12: 461-479.

[34] Pinel P, Le Clec'H G, van de Moortele PF, Naccache L, LeBihan D, Dehaene S. Event-related fMRI analysis of the cerebral circuit for number comparison. Neuroreport 1999; 10: 1473-1479.

[35] Le Clec'H G, Dehaene S, Cohen L, et al. Distinct Cortical Areas for Names of Numbers and Body Parts Independent of Language and Input Modality. NeuroImage 2000; 12(4): 381-391.

[36] Chochon F, Cohen L, van de Moortele P-F, Dehaene S. Differential contributions of the left and right inferior parietal lobules to number processing. J Cog Neurosci Lett 1999; 11: 617- 630.

[37] Isaacs EB, Edmonds CJ, Lucas A, Gadian DG. Calculation difficulties in children of very low birthweight: A neural correlate. Brain 2001; 124: 1701-1707.

[38] Molko N, Cachia A, Riviere D, et al. Functional and structural alterations of the intraparietal sulcus in a developmental dyscalculia of genetic origin. Neuron 2003; 40(4): 847-858.

[39] Ashcraft MH. Cognitive arithmetic: A review of data and theory. Cognition 1992; 44: 75-106.

[40] Campbell JID. Network interference and mental multiplication. J Exp Psychol: Learn Mem Cog 1987; 13: 109-123. 
[41] Siegler RS. Individual differences in strategy choices: Good students, Not-so-good students, and perfectionists. Child Dev 1988; 59: 833-851.

[42] Wechsler D. WISC III UK, ed. Sidcup. 1992, Kent: The Psychological Corporation.

[43] Sattler JM. Assessment of children's intelligence and special abilities. 1982, Boston, MA: Allyn and Bacon.

[44] Khomsi A. Epreuve d'évaluation de la compétence en lecture (LMC-R). ed. L.E.d.C.d.P. Appliquée. 1998, Paris.

[45] Lobrot M. Batterie d'épreuves pour mesurer la lecture et l'orthographe : Manuel. 1980, Paris: Editions et Application Psychologiques.

[46] Mousty P, Leybaert J. Evaluation des habilités de lecture et d'orthographe au moyen de BELEC: données longitudinales auprès d'enfants francophones testés en 2ème et 4ème années. Rev Eur Psychol App 1999; 49(4): 325-342.

[47] Geary DC, Hoard MK, Hamson CO. Numerical and arithmetical cognition: Patterns of functions and deficits in children at risk for a mathematical disability. J Exp Child Psychol 1999; 74(3): 213.

[48] Krueger LE, Hallford EW. Why $2+2=5$ looks so wrong: On the odd-even rule in sum verification. Mem $\operatorname{Cog} 1984 ; 12: 171-180$.

[49] Groen GJ, Parkman JM. A chronometric analysis of simple addition. Psychol Rev 1972; 79: 329-343

[50] Campbell JID, Tarling DPM. Retrieval processes in arithmetic production and verification. Mem Cog 1996; 24: 156-172.

[51] Geary DC. The problem-size effect in mental addition: Developmental and cross-national trends. Math Cog 1996; 2: 63-93.

[52] Geary DC, Brown SC. Cognitive addition: Strategy choice and speed-of-processing differences in gifted, normal, and mathematically disabled children. Dev Psychol 1991; 27(3): 398.

[53] Lépine R, Roussel JM, Fayol M. Résolution procédurale ou récupération en mémoire des additions et multiplications élémentaires chez les enfants? L'Année Psychol 2003; 103: 51-80.

[54] Snodgrass JG, Vanderwart M. A standardized set of 260 pictures: norms for name agreemen, image agreement, familiarity, and visual complexity. J Exp Psy-Learn Mem Cog 1980; 6(2): 174-215.

[55] Koontz KL, Berch DB. Identifying simple numerical stimuli: Processing inefficiencies exhibited by arithmetic learning disabled children. Mathe Cog 1996; 2(1): 1-23.

[56] Dehaene S, Dehaene-Lambertz G, Cohen L. Abstract representations of numbers in the animal and human brain. Trend Neurosci 1998; 21: 355-361.

[57] Zago L, Pesenti M, Mellet E, Crivello F, Mazoyer B, TzourioMazoyer N. Neural correlates of simple and complex mental calculation. NeuroImage 2001; 13(2): 314-327.
[58] Delazer M, Benke T. Arithmetic facts without meaning. Cortex 1997; 33: 697-710.

[69] Kaufmann L. More evidence for the role of the central executivein retrieving arithmetic facts - a case study of severe developmental dyscalculia. J Clin Exp Neuropsychol 2002; 24(3): 302-310.

[60] Benson DF, Geschwind N. Developmental Gerstmann syndrom. Neurology 1970; 20(3): 293-298.

[61] Kinsbourne M, Warrington EK. The developmental Gerstmann syndrom. Arch Neurol 1963; 8: 490-501.

[62] McCloskey M, Harley W, Sokol SM. Models of arithmetic fact retrieval: An evaluation in light of findings from normal and braindamaged subjects. J Exp Psychol: Learn Mem Cog 1991; 17: $377-$ 397.

[63] Pesenti M, Seron X, Van der Linden M. Selective impairment as evidence for mental organisation of arithmetic facts: BB, a case of preserved subtraction? Cortex 1994; 30: 661-671.

[64] Dehaene S, Spelke E, Pinel P, Stanescu R, Tsivkin S. Sources of mathematical thinking: Behavioral and brain-imaging evidence. Science 1999; 284(5416): 970-974.

[65] Venkatraman V, Ansari D, Chee MWL. Neural correlates of symbolic and non-symbolic arithmetic. Neuropsychologia 2005; 43(5): 744-753.

[66] Ischebeck A, Zamarian L, Egger K, Schocke M, Delazer M. Imaging early practice effects in arithmetic. NeuroImage 2007; 36(3): 993-1003.

[67] Grabner RH, Ansari D, Reishofer G, Stern E, Ebner F, Neuper C. Individual differences in mathematical competence predict parietal brain activation during mental calculation. NeuroImage 2007; 38(2): 346-356.

[68] Warrington EK, Cipolotti L. Word comprehension. The distinction between refractory and storage impairments. Brain 1996; 119: 611625.

[79] Kaufmann L, Lochy A, Drexler A, Semenza C. Deficient arithmetic fact retrieval--storage or access problem?: A case study. Neuropsychologia 2004; 42(4): 482.

[70] Robinson CS, Menchetti BM, Torgesen JK. Toward a two-factor theory of one type of mathematics disabilities. Learn Dis Res Prac 2002; 17(2): 81-89. 